\title{
Gonadal Hormones are Associated with Body Shape and Symptoms of Disordered Eating in Women
}

\author{
Jessica L. Bird , Kirsten A. Oinonen* and Dwight Mazmanian \\ Department of Psychology, Lakehead University, Health, Hormones, and Behaviour Laboratory, Thunder Bay, ON
}

Received: : October 10, 2015; Accepted: October 30, 2015; Published: November 07, 2015

*Corresponding author: Kirsten A. Oinonen PhD, Health, Hormones, and Behaviour Laboratory, Department of Psychology, Lakehead University, 955 Oliver Road, Thunder Bay, Ontario, P7B $5 E 1$ CANADA, tel:(807)343 8096; fax:(807)346 7734; E-mail: koinonen@lakeheadu.ca

\begin{abstract}
Objective: The aim of this study was to examine relationships between gonadal hormone levels (estradiol, progesterone, and testosterone), body shape, body mass index (BMI), and symptoms of disordered eating, in healthy young women.

Method: Fifty-two free-cycling women completed subscales from the Eating Disorder Inventory 3 (EDI-3; Body Dissatisfaction, Drive for Thinness, and Bulimia). Salivary samples and body measurements [BMI and waist-to-hip ratio (WHR)] were collected.

Results: Progesterone levels were positively correlated with Bulimia and Total Symptom scores, and were not correlated with measures of anxiety, depression, or BMI. After controlling for BMI, progesterone levels were also correlated with Body Dissatisfaction scores, and testosterone levels showed a trend toward a negative correlation with Drive for Thinness scores. Estradiol levels were positively correlated with BMI and WHR.
\end{abstract}

Conclusions: This study suggests links between gonadal hormone levels, body fat, body shape, and symptoms of disordered eating in a non-clinical female sample.

Keywords: Progesterone; Testosterone; Estradiol; Body dissatisfaction; Bulimia; BMI; Body Shape

\section{Introduction}

Gonadal hormones, such as estrogen, progesterone, and testosterone are believed to play an etiological role in eating disorders because of their relationships with food intake, appetite, weight, and body shape [1-3]. Research that has focused on the complex interactions between hormones and disordered eating has been conducted using several different methodologies (e.g., epidemiological, genetic, and menstrual cycle studies). One facet of this line of research involves examining the direct, or activational effects of circulating gonadal hormones on eating disorder (ED) symptoms. The examination of activational effects in non-clinical samples is important as it minimizes the influence of nutritional deficits on hormonal functioning and provides a greater understanding of factors relevant to the subclinical ED symptoms that precede full-blown eating disorders.
The association between hormones and binge eating was assessed by Klump and colleagues in a community sample [4]. A small group of normal BMI college women provided 65 days of saliva samples for estradiol and progesterone assay, as well as mood and binge-eating scale scores. After controlling for BMI and negative affect, increases in binge eating across the menstrual cycle were significantly correlated with decreased estradiol and increased progesterone. In a similar study, a small sample of healthy women provided 35 to 65 days of saliva samples as well as daily ratings for body dissatisfaction, drive for thinness, and dietary restraint [5]. Body dissatisfaction and drive for thinness peaked during the midluteal phase of the cycle (when progesterone is typically high), and both measures were negatively associated with estradiol but positively associated with progesterone. Except for the correlation between body dissatisfaction and estradiol, the associations showed little change after controlling for negative affect and emotional eating.

Therefore, there is evidence to suggest that both circulating estradiol and progesterone levels may be related to the severity of disordered eating behaviour in community samples. The purpose of the current study was to further examine the activational effects of gonadal hormones on ED symptoms in a non-clinical sample of women. The current study examined circulating levels of gonadal hormones and possible associations with Drive for Thinness, Bulimia, and Body Dissatisfaction scores on the EDI-3 [6]. A unique aspect of this study was the inclusion of measures of testosterone levels, in addition to measures of estradiol and progesterone. Studies have found associations between a diagnosis of bulimia nervosa and elevated testosterone in women [7-8]. Greater understanding of the contribution of hormones in eating behavior may have implications for prevention and treatment of disordered eating and obesity.

\section{Method}

Participants were recruited via email from a group of female university students who had completed an online questionnaire about women's health. Fifty-two women between the ages of 18 and 30 years $(M=20.56, S D=2.78)$, who were primarily 
of European descent (83\%) participated. Exclusion criteria included self-report of: current pregnancy or lactation, a current diagnosis of an eating or endocrine disorder, and the use of steroid medications (including hormonal contraceptives) in the past three months. All procedures were carried out with adequate understanding and written consent of the participants, and were approved by the university's research ethics board.

Participants were scheduled for a laboratory session that fell either during the follicular postmenses $(n=37)$ or midluteal phase $(n=15)$ of the cycle. Including women at two cycle phases allowed for an examination of the combined influence of both cyclical hormonal effects and individual difference hormonal effects. Samples in the postmenses phase were provided a mean of $1.94(S D=0.79)$ days after the last day of menses, while midluteal samples were provided a mean of $6.25(S D=1.06)$ days before the start of next menses. Using the forward count method, postmenses samples were provided on the mean cycle day $7.25(S D=1.36)$, and midluteal samples were provided on the mean cycle day $23.67(S D=2.06)$. Two samples, one from each phase, were excluded from analyses because they were provided outside the specified range of the menstrual cycle phases.

A digital scale was used to measure the participant's weight. Anthropometric tape was used to measure the height and the circumference of waist and hips. Body mass index [BMI; weight $(\mathrm{kg}) /$ height $\left.^{2}(\mathrm{~m})\right]$ and waist-to-hip ratio (WHR) were calculated using the above weight and height measurements. Participants had a mean BMI of $24.43(S D=8.72)$ and a mean WHR of 0.77 (SD $=0.08)$. Five participants $(9.6 \%)$ fell in the underweight range (BMI below 18.5), thirty-four (65.4\%) had BMIs in the normal range (between 18.5 and 24.9), and thirteen women (25.0\%) fell in the overweight/obese range (BMIs of 25.0 and above).

The Drive for Thinness, Bulimia, and Body Dissatisfaction subscales of the EDI-3 [6], as well as the total score of the three subscales (Total Symptom Score), were used to assess ED symptoms. An alternate scoring system was used in order to examine the full range of symptoms in a non-clinical population [9]. Reliability analyses with this scoring method for the Drive for Thinness, Bulimia, and Body Dissatisfaction scales with a sample of 642 young women yielded Cronbach's alphas of 0.86, 0.84 , and 0.92 , respectively. The Depression $(M=5.96, S D=$ 6.10) and Anxiety $(M=2.80, S D=4.01)$ scales of the Symptom Checklist-90-Revised (SCL-90-R) [10] were administered to assess participants' symptoms in the past week.

Levels of estradiol, progesterone, and testosterone were obtained by saliva sampling. Studies support the use of saliva sampling as a minimally invasive method which accurately detects the unbound biologically active form of the steroid molecules [11-12]. To control for circadian effects, salivary samples were collected between 8:30 AM and 10:30 AM, with sessions occurring a mean of 61 minutes ( $S D=30$ minutes) after participants awoke in the morning. Participants confirmed their menstrual cycle status and that they had refrained from eating, smoking, exercising, brushing teeth, or drinking anything but water since waking. To physically average the pulsatile secretion of hormones that occurs over time [13], participants drooled passively into collection tubes over the course of 45 minutes. Samples were stored in a freezer at $-34^{\circ} \mathrm{C}$ until they were shipped for radioimmunoassay analysis by Rocky Mountain Analyticals (Calgary, Alberta). For progesterone and testosterone, kits from DRG International Inc. (Germany) were utilized, and the estradiol kit was manufactured by IBL International (Germany). The detection limit was defined as that concentration for which the difference between duplicate samples exceeded $20 \%$, and was $0.8 \mathrm{pg} / \mathrm{mL}$ for estradiol, $20 \mathrm{pg} / \mathrm{mL}$ for progesterone, and 10 $\mathrm{pg} / \mathrm{mL}$ for testosterone. The intra-assay coefficients of variation (CVs) ranged from 10 to $15 \%$, and inter-assay CVs ranged from 7 to $10 \%$ at concentrations 3 to 4 times above the detection limit.

\section{Results}

\section{Descriptive Statistics and Preliminary Analyses}

Hormone values were examined in comparison to lower limits of detection and values below lower limits of detection were excluded [14]. Four values for estradiol were excluded, all from the postmenses phase. Thirty values for progesterone were excluded, with 26 of the ineligible values having been sampled in the postmenses phase. Two values were excluded for testosterone, one from each phase. After log transformation of the estradiol, BMI, depression, and anxiety values to achieve normality, no outliers were present. See Table 1 for means and standard deviations of all hormone assays. The mean hormone levels are similar to those found in other published studies of women in the same age range [14-15]. As expected, women in the two cycle phases differed in progesterone levels (midluteal > postmenstrual), $t(15)=-3.572, p=.003$; but not estradiol levels, $t(44)=-0.863, p=.393$. They did not differ in BMI, $t(50)=0.046$, $p=.963$; but did differ in WHR (midluteal > postmenstrual), $t(50)$ $=-2.152, p=.036$. Given the group $/$ phase differences in WHR and progesterone, associations between these two variables were not examined further.

\section{Main Analyses}

Pearson product-moment correlations between hormone values and laboratory body measurements were computed (see Table 1). Salivary estradiol was significantly positively correlated with BMI $(r=.364, p=.013, n=46)$ and WHR $(r=.405, p=.005, n$ =46). After controlling for BMI, the correlation between estradiol and WHR remained significant ( $r=.325, p=.029, n=43$ ). Body Dissatisfaction scores were significantly correlated with BMI ( $r=$ $.465, p=.001, n=52$ ), but Bulimia and Drive for Thinness scores showed no relationship with BMI (see Table 1). There were no other significant correlations between salivary hormone levels and variables such as participant age, time since waking, BMI, WHR, or Depression and Anxiety scores.

Correlations were examined for all three gonadal hormones and EDI-3 scores (see Table 1). There were no significant correlations between estradiol and any of the EDI-3 subscale scores (nor when controlling for BMI). However, progesterone levels were significantly positively correlated with Bulimia scores $(r=.591, p=.012, n=17)$, as well as the Total Symptom 
Table 1: Means and Standard Deviations for Hormone Salivary Assays and Correlations between Hormone Values, EDI-3 Subscale Scores, and Body Measurements.

\begin{tabular}{|c|c|c|c|c|c|c|}
\hline Variables [M $(S D), \mathrm{N}]$ & BU & BD & DT & Total & BMI & WHR \\
\hline $\begin{array}{l}\mathrm{E}_{2}[3.50(2.19), 46] \\
\mathrm{P}[59.00(37.24), 17] \\
\mathrm{T}[30.81(11.95), 48] \\
\operatorname{BMI}[24.43(8.72), 52]\end{array}$ & $\begin{array}{c}-.010 \\
.591^{*} \\
-.120 \\
.043\end{array}$ & $\begin{array}{l}.113 \\
.328 \\
-.065 \\
.465^{* *}\end{array}$ & $\begin{array}{l}.072 \\
.306 \\
-.237 \\
.175 \\
\end{array}$ & $\begin{array}{l}.065 \\
.524^{*} \\
-.170 \\
.252\end{array}$ & $\begin{array}{l}.364^{*} \\
-.252 \\
.203 \\
--\end{array}$ & $\begin{array}{c}.325^{* *} \\
\mathrm{a} \\
-.145 \\
.341^{*}\end{array}$ \\
\hline $\begin{array}{l}\text { Controlling for BMI } \\
\mathrm{E}_{2} \\
\mathrm{P} \\
\mathrm{T}\end{array}$ & $\begin{array}{c}-.0 .28 \\
.623^{* *} \\
-.131\end{array}$ & $\begin{array}{c}-.068 \\
.519 * \\
-.184\end{array}$ & $\begin{array}{l}.009 \\
.368 \\
-.282^{\mathrm{t}}\end{array}$ & $\begin{array}{c}-.029 \\
.628 * * \\
-.233\end{array}$ & $\begin{array}{l}-- \\
-- \\
--\end{array}$ & $\begin{array}{c}.302^{*} \\
\mathrm{a} \\
-.254\end{array}$ \\
\hline \multicolumn{7}{|c|}{$\begin{array}{l}\text { Note. Means and standard deviations presented are untransformed values. Correlations are presented in the top half of the table and partial } \\
\text { correlations are presented in the bottom half. } \mathrm{E}_{2}=\text { estradiol; } \mathrm{P}=\text { progesterone; } \mathrm{T}=\text { testosterone; } \mathrm{BU}=\mathrm{Bulimia} \text {; } \mathrm{BD}=\mathrm{Body} \text { Dissatisfaction; DT = Drive } \\
\text { for Thinness; Total = Total Symptom score (sum of BU, BD, DT scores); BMI = body mass index; WHR = waist-to-hip ratio. [waist circumference (cm) } \\
\text { / hip circumference }(\mathrm{cm})] \text {. } \\
{ }^{a} \text { Correlations not examined due to group/phase differences in progesterone and WHR. } \\
{ }^{\mathrm{t}} p<.06 .^{*} p<.05 .^{* *} p \leq .01 .\end{array}$} \\
\hline
\end{tabular}
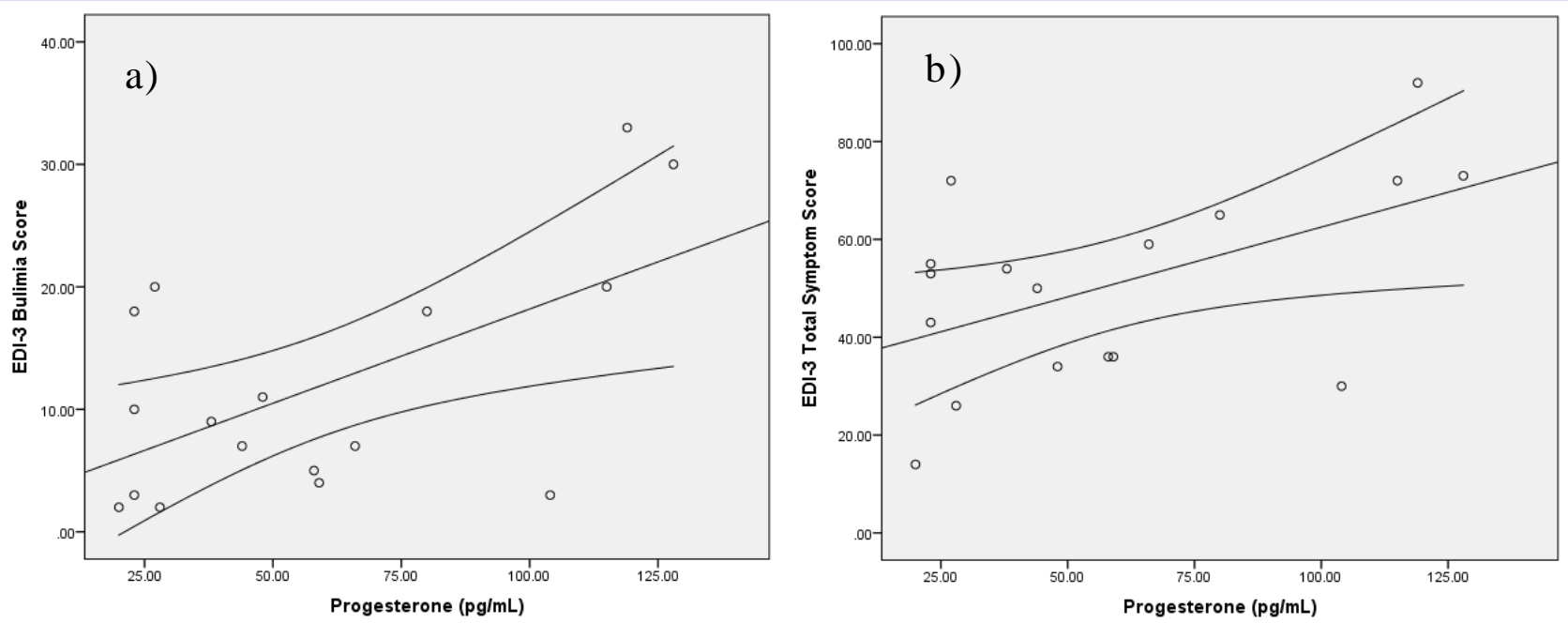

Figure 1: Scatterplot of progesterone levels and: (a) Total Symptom scores, and (b) EDI-3 Bulimia scores. Progesterone levels showed significant positive correlations with both Total Symptom scores (sum of Bulimia, Body Dissatisfaction, and Drive for Thinness scores) $(r=.524, p=.031, n=17$ ); and Bulimia scores $(r=.591, p=.012, n=17)$.

score $(r=.524, p=.031, n=17$ ) (see Figure 1). To ensure that these relationships were not due to the influence of body fat, partial correlations between hormone levels and EDI-3 scores while controlling for BMI were performed. After controlling for BMI, progesterone remained significantly positively correlated with the Bulimia ( $r=.623, p=.010, n=17)$ and Total Symptom $(r$ $=.628, p=.009, n=17$ ) scores; and was now positively associated with Body Dissatisfaction ( $r=.519, p=.039, n=17$ ) subscale scores. When controlling for BMI, there was a trend toward a negative correlation between testosterone levels and Drive for Thinness scores ( $r=-.282, p=.055, n=48)$.

\section{Discussion}

The findings of the current study provide further support for a relationship between circulating hormone levels and symptoms of disordered eating in a community sample of women. Progesterone levels were positively correlated with Bulimia scores and Total Symptom Scores (even after controlling for
BMI). The effect sizes for the relationships between progesterone and EDI-3 scores were in the large range (e.g., small, medium, and large effect sizes are .10, .30, and .50, respectively) [16]. These findings are consistent with menstrual cycle studies that have found increased bulimic symptoms during the latter half of the cycle, including the midluteal phase when progesterone levels are peaking and estrogen levels are moderately high, and the premenstrual phase when both estrogen and progesterone levels are falling [4, 17-18]. Two studies have also found positive correlations between progesterone and increases in binge eating in both clinical and non-clinical samples $[4,11]$. The positive association between progesterone and bulimia symptoms is also consistent with findings of increased appetite during natural periods of elevated progesterone, such as the luteal phase of the menstrual cycle, and during pregnancy and lactation [2]. These changes in appetite are believed to have adaptive value.

After controlling for BMI, progesterone was also significantly positively correlated with Body Dissatisfaction scores. A small 
number of studies have examined body dissatisfaction in relation to gonadal hormones. Altabe and Thompson found greater body image dissatisfaction and greater dissatisfaction with physical appearance in the premenstrual phase [20]. Racine and colleagues examined daily ratings of disordered eating symptoms across the menstrual cycle while controlling for negative affect and emotional eating [5]. Body dissatisfaction showed a pattern of fluctuation across the menstrual cycle, with significantly higher levels during the midluteal/premenstrual phases compared to the follicular/ovulatory phases. Given higher levels of progesterone in midluteal/premenstrual cycle phases, the present findings are consistent with these two previous studies.

An additional finding of the current study was that testosterone showed a trend toward a negative correlation with Drive for Thinness scores, after controlling for BMI. There are few studies to date that have examined the relationship between testosterone levels and eating disorder symptoms, and the current study appears to be the first to examine the relationship between testosterone and Drive for Thinness in healthy women. Most studies have examined hormone levels in groups of women diagnosed with eating disorders, and suggest that elevated testosterone levels may be associated with bulimia nervosa [8], and decreased testosterone levels may be found in women with anorexia nervosa [21]. Our finding supports further exploration of the relationship between testosterone levels and eating disorder symptoms in non-clinical samples.

While noteworthy findings emerged for circulating levels of progesterone, no significant findings were noted for estradiol levels and symptoms of disordered eating. The small sample size in this study and small range of estradiol levels (compared to the larger range of levels for progesterone and testosterone) may have limited the ability to detect associations between estradiol levels and symptoms of disordered eating. Estradiol levels were not correlated with EDI-3 scores in this study, however there was a positive correlation between estradiol and both BMI and WHR, suggesting medium effect sizes. Previous findings with regard to the correlation between estradiol and BMI in healthy premenopausal women are inconsistent, but overall, research tends to suggest the absence of a relationship [22]. One study that examined WHR and gonadal hormones in healthy premenopausal women found no relationship [23], while another study found that women with higher WHR $(>.80)$ had higher salivary estradiol levels across the menstrual cycle [24]. Further exploration of these relationships is warranted. Given that both estradiol and progesterone levels demonstrated correlations with WHR, body shape may be an additional factor to consider in studies examining hormones and symptoms of disordered eating.

Overall, the findings of the current study provide additional evidence for a relationship between gonadal hormones, specifically progesterone, and symptoms of disordered eating across the menstrual cycle. While the body of research in this area indicates that relationships are complex, this and other studies suggest that gonadal hormones may influence symptoms of disordered eating, independent of levels of anxiety, depression, and body shape or size. The results of the study support the growing body of research examining genetic and biological factors relevant to the development of eating disorder symptoms. The findings also suggest a relationship between gonadal hormones and body weight and shape, which may be relevant for understanding biological factors related to obesity and both treatment and prevention.

Limitations of the current study included self-report of eating disorder status and the small sample size. Also, the inclusion of women at two menstrual cycle phases has both strengths and weaknesses but allowed for an examination of the combined influence of both cyclical hormonal effects and individual difference hormonal effects on WHR, BMI, and symptoms of disordered eating. Future studies should replicate these findings both across the menstrual cycle and within specific cycle phases. The use of a non-clinical sample is a strength, as it adds to knowledge of symptoms of disordered eating in the general population, and minimizes the possible effects of severe disordered eating behaviours on the hormonal functioning of participants. Additional strengths of the study include the sampling of hormones over a 45-minute period to control for pulsatile hormone release; and the inclusion of measures of anxiety, depression, and body shape. Finally, this is the first study to examine levels of testosterone, progesterone, and estradiol in relation to symptoms of disordered eating in one non-clinical sample.

\section{Acknowledgements}

Some of the work presented here was based on a $\mathrm{PhD}$ dissertation submitted by J.L.B. to Lakehead University. J.L.B. is now with IWK Health Centre (Halifax, Canada). To conduct this research, J.L.B. received an Ontario Women's Health Council / Institute of Gender and Health Doctoral Research Award from the Canadian Institute of Health Research. Additional funding was awarded to K.A.O. (Principal Investigator) and J.L.B. (Collaborator) by the Social Science and Humanities Research Council of Canada Research Development Fund. We would like to acknowledge Dr. Ron Davis (Lakehead University), Dr. Vicki Kristman (Lakehead University), and Dr. Jennifer Mills (York University) for their helpful comments and suggestions on an earlier draft. We would also like to thank Nicole Westlund and Courtney Brown for help with data collection.

\section{References}

1. Brown LM, Gent L, Davis K, Clegg DJ. Metabolic impact of sex hormones on obesity. Brain Res. 2010;1350:77-85, doi: 10.1016/j. brainres.2010.04.056.

2. Hirschberg AL. Sex hormones, appetite and eating behaviour in women. Maturitas. 2012; 71(3):248-56, doi: 10.1016/j. maturitas.2011.12.016.

3. Steiner M, Dunn, E, Born, L. Hormones and mood: from menarche to menopause and beyond. Journal of Affect Disorders. 2003;74(1):6783.

4. Klump KL, Keel PK, Culbert KM, Edler C. Ovarian hormones and binge eating: Exploring associations in community samples. Psychol Med. 2008;38(12):1749-1757, doi: 10.1017/S0033291708002997.

5. Racine SE, Culbert KM, Keel PK, Sisk CL, Burt SA, Klump KL. Differential 
associations between ovarian hormones and disordered eating symptoms across the menstrual cycle in women. Int J Eat Disorder. 2012;45(3):333-344, doi:10.1002/eat.20941.

6. Garner DM.. Eating disorder inventory-3 (EDI-3). Lutz, FL: Psychological Assessment Resources, Inc.; 2004.

7. Cotrufo P, Monteleone P, d'Istria M, Fuschino A, Serino I, Maj M Aggressive behavioral characteristics and endogenous hormones in women with bulimia nervosa. Neuropsychobiology. 2000;42(2):58 61.

8. Sundblad C, Bergman L, Eriksson E. High levels of free testosterone in women with bulimia nervosa. Acta Psychiat Scand.1994; 90(5):397398.

9. Bird JL, Oinonen, KA. Elevated eating disorder symptoms in women with a history of oral contraceptive side effects. Arch Women Ment Hlth. 2011;14(4):345-53, doi:10.1007/s00737-011-0229-z.

10. Derogatis LR. Symptom checklist-90-R (SCL-90-R): Administration, scoring, and procedures manual 3ed. Minneapolis, MN: National Computer Systems Inc; 1994.

11. Edler C, Lipson SF, Keel PK. Ovarian hormones and binge eating in bulimia nervosa. Psychol Med. 2007;37(1):131-41.

12. Shirtcliff EA, Granger DA, Schwartz EB, Curran MJ, Booth A, Overman WH. Assessing estradiol in biobehavioural studies using saliva and blood spots: Simple radioimmunoassay protocols, reliability, and comparative validity. Horm Behav. 2000; 38(2):137-47.

13. Brambilla DJ, O’Donnell AB, Matsumoto AM, McKinley JB. Intraindividual variation in levels of serum testosterone and other reproductive and adrenal hormones in men. Clin Endocrinol 2007;67(6):853-62.

14.Griksiene R, Ruksenas, O. Effects of hormonal contraceptives on mental rotation and verbal fluency. Psychoneuroendocrino. 2011;36(8):1239-1248,doi:10.1016/j.psyneuen.2011.03.001.

15. Liening SH, Stanton SJ, Saini E, Schultheiss OC. Salivary testosterone, cortisol, and progesterone: Two-week stability, interhormone correlations, and effects of time of day, menstrual cycle, and oral contraceptive use on steroid hormone levels. Physiol Behav. 2010;99(1):8-16, doi: 10.1016/j.physbeh.2009.10.001.

16. Cohen J. A power primer. Psychol Bull. 1992;112(1):155-159. doi: 10.1037/0033-2909.112.1.155.

17. Gladis MM, Walsh BT. Premenstrual exacerbation of binge eating in bulimia. Am J Psychiat. 1987;144(12):1592-5.

18. Lester NA, Keel PK, Lipson SF. Symptom fluctuation in bulimia nervosa: Relation to menstrual-cycle phase and cortisol levels. Psychol Med. 2003;33(1):51-60.

19. Price WA, Torem MS, DiMarzio LR. Premenstrual exacerbation of bulimia. Psychosomatics. 1987;28(7):378-379.

20. Altabe M, Thompson JK. Menstrual cycle, body image, and eating disturbance.IntJEatDisorder.1990;9(4):395-401,DOI:10.1002/1098108X(199007)9: 4<395::AID-EAT2260090405>3.0.CO;2-E.

21. Monteleone P, Luisi M, Colurcio B, Casarosa E, Monteleone P, Ioime R, et al. Plasma levels of neuroactive steroids are increased in untreated women with anorexia nervosa or bulimia nervosa. Psychosom Med. 2001;63(1):62- 68 .

22. Key TJ, Allen NE, Verkasalo PK, Banks E. Energy balance and cancer: The role of sex hormones. P Nutri Soc. 2001;60(1):81-9.

23. Tworoger SS, Eliassen AH, Missmer SA, Baer H, Rich-Edwards J, Michels KB, et al. Birthweight and body size throughout life in relation to sex hormones and prolactic concentrations in premenopausal women. Cancer Epidem Biomar. 2006;15(12):2494-2501.

24. Emaus A, Espetvedt S, Veierod MB, Ballard-Barbash R, Furberg AS, Ellison PT, et al. 17-beta-estradiol in relation to age at menarche and adult obesity in premenopausal women. Hum Reprod. 2008;23(4):919-927, doi: 10.1093/humrep/dem432. 